\title{
Polarized Drell-Yan Experiments at RHIC and J-PARC
}

\section{Yuji Goto}

RIKEN Nishina Center for Accelerator-Based Science, Wako, Saitama 351-0198, Japan RIKEN BNL Research Center, Brookhaven National Laboratory, Upton, New York 11973, USA

E-mail: goto@bnl.gov

\begin{abstract}
We present physics motivations and experimental consideration for a fixed-target polarized DrellYan dimuon experiments at RHIC and at J-PARC. Dimuons from the Drell-Yan process are detected using a spectrometer based on the existing Fermilab E906/SeaQuest spectrometer. Through the detections of the Drell-Yan events, a broad physics program addressing various issues in QCD is pursued. The primary goal of the proposed experiments is to extract the Sivers parton distribution function in the valence-quark region from the measurement of the single-spin asymmetry. The sensitivity of the proposed fixed-target experiments will provide a stringent test of the QCD prediction that the Sivers function in the Drell-Yan process has an opposite sign to that in deepinelastic scattering. Many other rich programs of spin-dependent structure function physics are carried out through the measurements of unpolarized, singly polarized, and doubly polarized Drell-Yan processes.
\end{abstract}

XVIII International Workshop on Deep-Inelastic Scattering and Related Subjects, DIS 2010 April 19-23, 2010

Firenze, Italy 


\section{Introduction}

The Drell-Yan process, in which a pair of charged leptons are produced in quark-antiquark annihilation, is an ideal tool for probing parton distributions inside the proton. The mechanism of the Drell-Yan process is well understood and the absence of hadronization processes in the final state eliminates the uncertainties caused by the relatively poorly known fragmentation functions encountered in semi-inclusive DIS process or hadron production in $\mathrm{p}-\mathrm{p}$ collisions.

A transverse-structure measurement with transversely-polarized beams is an actively developing subject to understand the proton structure multidimensionally. It extends our understanding of the collinear structure of the proton, and give us information of orbital angular momentum in the proton and shape of the proton. One of the frameworks to understand the structure of the proton is the transverse-momentum dependent (TMD) distribution. A single-spin asymmetry (SSA) measurement of the Drell-Yan process will give a clear measurement of one of the TMD distribution functions, Sivers function [1]. The Sivers function represents a correlation between proton transverse spin and parton transverse momentum, and it is closely related to the orbital angular momentum in the proton. In order to measure the Sivers function from the SSA, the measurement process needs to have an initial-state or final-state interaction with remnant partons in the proton. The deep-inelastic scattering process in the lepton scattering has a final-state interaction and the Drell-Yan process in the hadron reaction has an initial-state interaction with remnant partons in the proton. The TMD framework predicts the Sivers function measured by both processes should have the opposite sign each other [2]. It is a stringent test of the TMD framework to investigate this property of the opposite sign with use of both the lepton scattering and the hadron reaction.

By using either of the transversely polarized beam or target, the SSA measurement gives the transversity distribution combined with the Boer-Mulders function. This measurement and the Sivers function measurement are distinguished by different angular distribution. The transversity distribution shows a transverse-spin distribution of a parton inside the transversely polarized proton. The Boer-Mulders function shows a correlation between the transverse-spin and the transverse momentum of a parton inside the unpolarized proton. The Boer-Mulders function is also measured by the angular distribution of the unpolarized Drell-Yan measurement.

The transversity parton distributions can also be measured in polarized Drell-Yan experiments with a transversely polarized proton beam colliding with either another transversely polarized proton beam or target. The double transverse spin asymmetry is proportional to the product of the transversity distribution of the quark and that of the antiquark [3]. Such measurements would lead to the determination of the antiquark transversity distributions which are difficult to isolate from the semi-inclusive DIS experiments. It is worth noting that information on the antiquark transversity distributions are required to determine the nucleon tensor charge, for which lattice QCD calculations are already available [4].

With spin rotator magnets which makes the beam longitudinally polarized, the flavor asymmetry measurement of the sea-quark polarization can be measured. To obtain the total quark and gluon contribution to the nucleon spin with high precision, it is important to know the flavor-sorted sea-quark contribution to the nucleon spin directly. Because the $Q^{2}$ evolution of the quark and gluon distribution mixes them, uncertainties of the flavor-sorted sea-quark distribution propagate to uncertainties of the quark and gluon distributions. 


\section{Polarized Drell-Yan Experiments}

We have proposed polarized Drell-Yan experiments using polarized proton beams and fixed targets at RHIC and J-PARC. Dimuons from the Drell-Yan process are detected using a spectrometer based on the existing Fermilab E906/SeaQuest spectrometer. The SeaQuest spectrometer comprises a solid iron focusing dipole magnet containing the beam dump, followed by a large open aperture dipole for precise momentum determination. Multi-wire proportional and drift chambers between the first two magnets as well as after the second dipole provide tracking information, supplemented by scintillation hodoscopes used primarily for fast triggering. A final large iron absorber instrumented with proportional tubes provides muon identification. We expect to use as many of the detector elements and electronics as possible from the SeaQuest experiment available after the completion of the experiment in 2013.

At RHIC, we have proposed a measurement of dimuons from Drell-Yan process with polarized proton beams and an internal target at the IP2 area [5]. It is a big advantage that we can use $250 \mathrm{GeV}$ polarized proton beams which is currently operational for collider experiments. At J-PARC, we have proposed the acceleration of polarized proton beams to $30-50 \mathrm{GeV}$ [6]. The polarized-proton beam is extracted to the high-momentum beam line, and used with polarized targets. Through the detections of the Drell-Yan events with various targets, a broad physics program addressing various issues in QCD is pursued.

There are proposals of polarized Drell-Yan measurements at RHIC by existing collider experiments (STAR and PHENIX) and a dedicated collider experiment [7] which are not discussed in this article.

\subsection{RHIC}

Using $250 \mathrm{GeV}$ transversely polarized proton beams and an internal target at the IP2 area, dimuons from the Drell-Yan process can be detected using a spectrometer based on the SeaQuest spectrometer. The primary goal of the proposed experiment is to extract the Sivers parton distribution functions in the valence-quark region from the measurement of the Drell-Yan single-spin asymmetry.

The IP2 area (previous BRAHMS site) is the candidate experimental site. It has an available space of $\pm 7 \mathrm{~m}$ of the nominal interaction point (or $14 \mathrm{~m}$ in total). We request to have two phases of the beam-time, parasitic beam time with collider experiments as a phase-1, and dedicated beam time as a phase- 2 . In the phase-1, we request a beam intensity of $2 \times 10^{11}$ with $\sim 10 \mathrm{MHz}$, and a cluster-jet target or pellet target with a thickness of $10^{15}$ atoms $/ \mathrm{cm}^{2}$. We hope it is acceptable as a parasitic experiment with collider experiments. It corresponds to a luminosity of $2 \times 10^{33} \mathrm{~cm}^{-2} \mathrm{~s}^{-1}$. We can accumulate $10,000 \mathrm{pb}^{-1}$ luminosity with $5 \times 10^{6}$ seconds. In the phase- 2 , we request a beam intensity of $2 \times 10^{11}$ with $\sim 15 \mathrm{MHz}$, and a pellet target or solid target with a thickness of $10^{16}$ atoms $/ \mathrm{cm}^{2}$. It corresponds to a luminosity of $3 \times 10^{34} \mathrm{~cm}^{-2} \mathrm{~s}^{-1}$. We can accumulate $30,000 \mathrm{pb}^{-1}$ luminosity with $10^{6}$ seconds.

A fast Monte Carlo simulation with an experimental apparatus fitted in the IP2 area has been performed to evaluate the kinematic acceptance and yield of the experiment. Results of the fast Monte Carlo simulation with PYTHIA for the internal-target experiment at $E_{\text {beam }}=250 \mathrm{GeV}$ or $\sqrt{s}=22 \mathrm{GeV}$ are shown. By using a transversely polarized beam, measurement of single-spin 

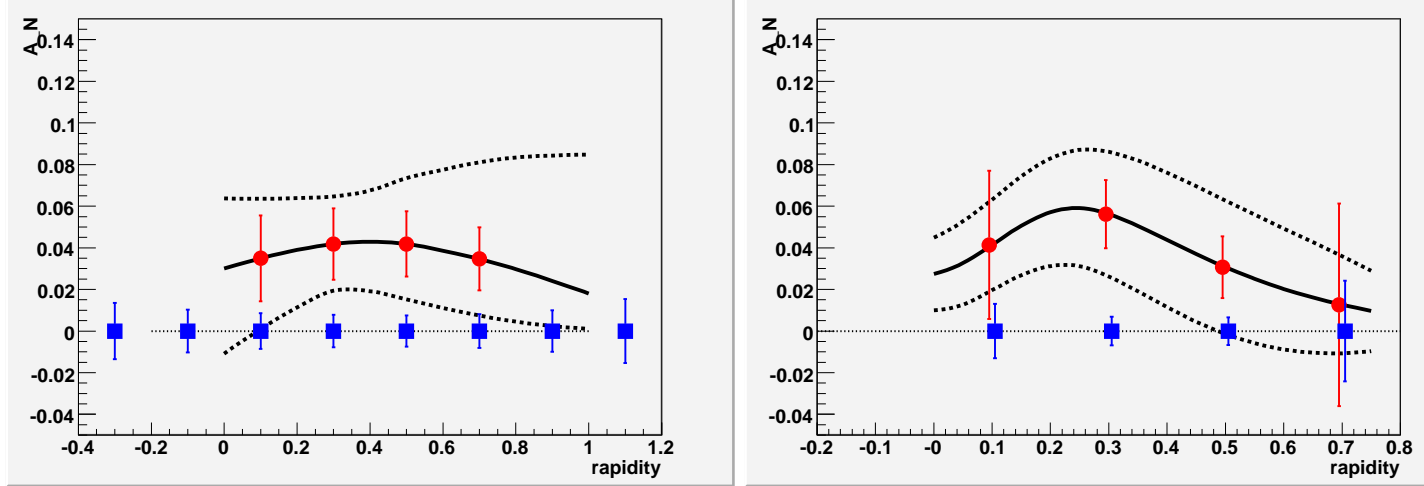

Figure 1: Left: Drell-Yan $A_{N}$ integrated over $0<q_{T}<1 \mathrm{GeV} / c$ and $4.5<M_{\mu^{+} \mu^{-}}<8 \mathrm{GeV}$ at RHIC. Expected statistical sensitivities for $10,000 \mathrm{pb}^{-1}$ (red circles) in phase-1 and $40,000 \mathrm{pb}^{-1}$ (blue squares) in phase-1 + phase-2 are shown. Right: Drell-Yan $A_{N}$ integrated over the transverse momentum of the virtual photon and dimuon mass range $4<M_{\mu^{+} \mu^{-}}<5 \mathrm{GeV}$ at J-PARC. Expected statistical sensitivities are shown for a 120-day measurement with unpolarized liquid $\mathrm{H}_{2}$ target (red circles) and nuclear target (blue squares).

asymmetry, or $A_{N}$ :

$$
A_{N}=\frac{\sigma_{L}-\sigma_{R}}{\sigma_{L}+\sigma_{R}}
$$

gives the Sivers function. The left panel of Figure 1 shows the $A_{N}$ integrated over transverse momentum $0<q_{T}<1 \mathrm{GeV} / c$ of the virtual photon and dimuon mass range $4.5<M_{\mu^{+} \mu^{-}}<8$ $\mathrm{GeV}$ calculated with the Sivers function [8, 9]. Expected statistical sensitivities are calculated for $10,000 \mathrm{pb}^{-1}$ in phase- 1 and $40,000 \mathrm{pb}^{-1}$ in phase- $1+$ phase- 2 assuming $70 \%$ polarization. By these measurements, we will be able to study not only the sign of the Sivers function but also the shape of the function.

The sensitive $x$ region of the Sivers function measurement is 0.2 - 0.6 which has an overlap with the measured region by the DIS experiments $(x<0.3)$ and cover higher- $x$ region with better sensitivity. We want to make the $x$-coverage as low as possible to compare with the DIS data with better sensitivity.

\subsection{J-PARC}

J-PARC is a high-intensity proton accelerator facility at Tokai in Japan. We have proposed the construction of a spectrometer at J-PARC designed for the detection of high-mass dimuons produced in the interactions of $50 \mathrm{GeV}$ primary proton beams with various targets [10]. Through the detections of the Drell-Yan events, a broad physics program addressing various issues in QCD is pursued. We have also proposed the acceleration of polarized proton beams to $30-50 \mathrm{GeV}$ at the J-PARC facility [6]. Polarized proton beam acceleration with two partial helical Siberian snakes is shown to be feasible in discussion with J-PARC and BNL accelerator physicists.

By using a transversely polarized beam, the SSA measurement gives the Sivers function. We request to use $5 \times 10^{12}$ ppp beam with unpolarized liquid $H_{2}$ target, or nuclear target. The right panel of Figure 1 shows the SSA integrated over the transverse momentum of the virtual photon and dimuon mass range $4<M_{\mu^{+} \mu^{-}}<5 \mathrm{GeV}$ calculated with Sivers function [11, 12]. Expected 
statistical sensitivities are calculated by PYTHIA simulation for a 120-day measurement assuming $50 \%$ duty factor and $75 \%$ polarization. Red points show the sensitivity with $51-\mathrm{cm}$-long (5\% interaction length) unpolarized liquid $H_{2}$ target located at $127-\mathrm{cm}$ upstream from the entrance of the analyzing magnet. Blue points show that with $20 \%$-interaction length nuclear target located at just downstream of the $H_{2}$ target.

A rich program of spin physics can be carried out through the measurements of unpolarized, singly polarized, and doubly polarized Drell-Yan processes. Measurement of the orbital angular momentum component in the nucleon is one of the most important goal of the spin physics program of the J-PARC dimuon experiment.

\section{Acknowledgments}

The author would like to thank people on the proposals, especially J. C. Peng, S. Sawada, H. Sato, P. E. Reimer and D. F. Geesaman, and is grateful to U. D'Alesio, S. Melis, F. Yuan, and W. Vogelsang for discussions and theory calculations.

\section{References}

[1] D. W. Sivers, Phys. Rev. 41, 83 (1990).

[2] S. J. Brodsky, D. S. Hwang and I. Schmidt, Phys. Lett. 530, 99 (2002); Nucl. Phys. B 642, 344 (2002); J. C. Collins, Phys. Lett. B 536, 43 (2002); X. Ji and F. Yuan, Phys. Lett. B 543, 66 (2002);

A. V. Belitsky, X. Ji and F. Yuan, Nucl. Phys. B 656, 165 (2003); D. Boer, P. J. Mulders and F. Pijlman, Nucl. Phys. B 667, 201 (2003).

[3] J. P. Ralston and D. E. Soper, Nucl. Phys. B 152, 109 (1979).

[4] M. Gockeler et al. [QCDSF Collaboration and UKQCD Collaboration], Phys. Lett. B 627, 113 (2005) [arXiv:hep-lat/0507001].

[5] http://www.bnl.gov/npp/docs/pac0610/Goto_rhic-drell-yan.pdf

[6] http://j-parc.jp/NuclPart/pac_0801/pdf/Goto.pdf

[7] http://www.bnl.gov/npp/docs/pac0610/Crawford_LoI.100524.v1.pdf

[8] M. Anselmino, M. Boglione, U. D’Alesio, S. Melis, F. Murgia and A. Prokudin, Phys. Rev. D 79, 054010 (2009) [arXiv:0901.3078 [hep-ph]].

[9] U. D'Alesio and S. Melis, private communications.

[10] http://j-parc.jp/NuclPart/pac_0606/pdf/p04-Peng.pdf

[11] X. Ji, J. Qiu, W. Vogelsang and F. Yuan, Phys. Rev. Lett. 97, 082002 (2006): Phys. Rev. D 73, 094017 (2006).

[12] F. Yuan and W. Vogelsang, private communications. 